\title{
Efeito da Modelagem probabilística dos ganhos nos lóbulos laterais das antenas das estações terrenas no cálculo de interferências entre sistemas via satélite
}

\author{
Alfredo Córdova, Jose Mauro P. Fortes.
}

\begin{abstract}
Resumo-Como alternativa ao procedimento usual de cálculo de interferências que utiliza diagramas de referência na caracterização dos ganhos das antenas, este trabalho propõe um procedimento mais próximo da realidade no qual os ganhos nos lóbulos laterais das antenas envolvidas são modelados por variáveis aleatórias. Neste caso, a razão portadora interferência resultante é também uma variável aleatória. Expressões analíticas para a função distribuição de probabilidade da razão são desenvolvidas para dois tipos de modelagem dos ganhos nos lóbulos laterais das antenas: como variáveis aleatórias com distribuição exponencial e como variáveis aleatórias com distribuição gama. Estas expressões são aplicadas a situações específicas envolvendo multiplos satélites multifeixe e os resultados obtidos são comparados àqueles resultantes do cálculo que utiliza um diagrama de referência na caracterização dos ganhos das antenas.
\end{abstract}

Palavras-Chave-Sistemas via Satélite; Interferência; Ganhos de Antenas; Modelagem Probabilística.

Abstract-As an alternative to the usual interference calculation procedure that uses a reference radiation pattern for antenna gain characterization, this work proposes a more realistic procedure in which the side lobe antenna gains are modeled as random variables. In this case the carrier to interference ratio is also a random variable. Analytical expressions for the probability distribution function of this ratio are determined for two types of modeling of the antenna sidelobe gains: as exponentially distributed random variables and as gamma distributed random variables. These analytical expressions are applied to specific situations involving multiple multi-beam satellites and the obtained results are compared to those resulting from the use of a reference antenna pattern in interference calculation.

Keywords-Satellite Systems; Interference; Antenna Gain; Probabilistic Modelling

\section{INTRODUÇÃO}

Usualmente, o cálculo de interferências em um ambiente envolvendo diversas redes de comunicação por satélite, considera aspectos associados a situações de pior caso. Situações de pior caso incluem, por exemplo, o posicionamento das estações terrenas transmissora e receptora dos diversos sistemas, a consideração de um diagrama de referência para o diagrama de radiação das antenas das estações terrenas, efeitos da atenuação por chuvas apenas no sinal desejado, entre outros.

$\mathrm{O}$ presente trabalho diz respeito à situação referente aos ganhos das antenas das estações terrenas em seu lóbulos laterais, usualmente tomados iguais aos valores de um diagrama de referência como, por exemplo, o diagrama de radiação

Alfredo Córdova, Jose Mauro P. Fortes. Centro de Estudos em Telecomunicações da PUC-Rio, Rio de Janeiro, Brasil, E-mails: acordova@cetuc.puc-rio.br, jmfortes@cetuc.puc-rio.br. em [1], onde o ganho nos lóbulos laterais da antena de uma estação terrena em uma direção formando um ângulo $\lambda$ com a direção de máxima radiação dado por $g(\lambda)=32-25 \log (\lambda)$. Esta expressão representa uma aproximação para uma curva obtida a partir de dados experimentais, e que corresponde a valores de ganhos excedidos por $10 \%$ dos picos. Para cada valor de $\lambda$, estes níveis correspondem à média dos valores obtidos para diversas antenas, cada um deles representando um nível excedido por $10 \%$ dos picos dentro de um intervalo angular especificado (por exemplo, 1 grau).

Obviamente, numa situação envolvendo múltiplas redes interferentes, considerar que em todas as redes interferentes os ganhos das antenas das estações terrenas na direção dos receptores do enlace do sistema vítima (enlaces de subida e descida) é igual aos ganhos dados pelo diagrama de referência utilizado constitui uma situação de pi or caso, uma vez que possivelmente nem todos os ganhos correspondem aos picos dos lóbulos laterais das antenas.

De modo a evitar esta situação de pior caso, o presente trabalho modela os ganhos nos lóbulos laterais das antenas das estações terrenas das diversas redes interferentes como variáveis aleatórias. A ideia de modelar estes ganhos como variáveis aleatórias surgiu por volta de 1976 e motivou o desenvolvimento de estudos iniciais $[2,3,4]$ nos quais o objetivo principal era determinar quais as funções densidade de probabilidade mais adequadas para modelar os ganhos nos lóbulos laterais das antenas. Em [2], por exemplo, foi proposto que o ganho de amplitude (expresso como fator) nos lóbulos laterais das antennas fosse modelado por uma variável aleatória de Rayleigh, com parâmetro a ser determinado. Como outra opção, o estudo em [3] analisou o efeito de modelar os ganhos de potência nos lóbulos laterais (expressos em fatores) como variáveis de Rayleigh para uma situação simples envolvendo apenas duas entradas de interferência. Como em [2], a análise em [4] considerou que o ganho de amplitude tem função densidade de probabilidade de Rayleigh e, consequentemente, os ganhos de potência (em fatores) são modelados por vari áveis aleatórias com função densidade de probabilidade exponencial. No estudo a densidade de probabilidade de Rayleigh é obtida como uma situação limite da densidade de probabilidade de Rice, inicialmente obtida para o ganho de amplitude com base no Teorema do Limite Central e, neste caso, o ganhos de potência teriam densidades de probabilidade de uma variável aleatória Rice ao quadrado.

Um estudo bastante completo sobre a escolha da densidade de probabilidade mais adequada para modelar os ganhos nos 
lóbulos laterais das antenas foi elaborado para o Intelsat, e seu relatório final [5] inclui a análise de dados de mais de 300 antenas. Com base nestes dados experimentais foram avaliadas as possibilidades de utilização de quatro possíveis densidades de probabilidade: exponencial, Rayleigh, Rice, Rice ao quadrado e Gama. A motivação para avaliar as densidades exponencial e Rice ao quadrado foi o estudo realizado em [4]. A consideração da distribuição Gama decorreu da observação de que, para quase todos os dados experimentais considerados, o desvio padrão excede a média, o que não acontece com as três primeiras densidades de probabilidade consideradas.

Os resultados destes trabalhos iniciais, motivaram alguns estudos envolvendo múltiplas redes interferentes [6,7,8]. Nestes estudos, os ganhos nos lóbulos laterais das antenas das estações terrenas foram modelados por variáveis aleatórias exponenciais, estatisticamente independentes e a escolha por esta modelagem deveu-se à possibilidade de se expressar analiticamente a função densidade de probabilidade de uma combinação linear de variáveis aleatórias estatisticamente independentes e com densidades de probabilidade exponenciais. Embora a análise em [5] tenha indicado que a densidade de probabilidade Gama fosse mais adequada, nenhum destes estudos considerou esta possibilidade.

No presente trabalho, tanto funções densidade de probabilidade exponenciais quanto gama são consiradas na modelagem dos ganhos nos lóbulos laterais das estações terrenas. A moldelagem matemática utilizada, que permitiu a obtenção de expressões analíticas para a função densidade de probabilidade da razão portadora-interferência, é apresentada na Seção II. $\mathrm{Na}$ Seção III as expressões desenvolvidas são utilizadas na análise de situações onde sistemas de satélites geoestacionários compartilham uma mesma faixa de freqüências. Mais especificamente, são analisados três cenários envolvendo, respectivamente, dois, três e cinco sistemas multi-feixe operando na Banda Ka. Finalmente as conclusões decorrentes dos resultados obtidos são apresentadas na Seção IV.

\section{Modelagem Matemática}

Nesta seção são obtidas expressões para as razões portadorainterferência num ambiente envolvendo múltiplos sistemas via satélite e expressões analíticas que permitem calcular a função densidade de probabilidade da razão portadora-interferência a partir das funções densidade de probabilidade dos ganhos das antenas das estações terrenas envolvidas. Estas expressões são particularizadas para as duas modelagens dos ganhos nos lóbulos laterais das antenas: Caso Exponencial e Caso Gama. Da geometria da Figura 1, observa-se que a razão portadorainterferência no lance de subida relativa à interferência do sistema $S_{j}$ sobre o sistema vítima $S_{V}$ pode ser escrita como

$$
\left(\frac{C}{I}\right)_{u p}=\frac{P_{1} g_{1}(0) g_{2}(\phi) \ell_{3 j}^{\prime}}{P_{1 j}^{\prime} g_{2}\left(\rho_{j}\right) g_{1 j}^{\prime}\left(\theta_{j}\right) \ell_{1}}
$$

onde $P_{1}, P_{1 j}^{\prime}$ representam respectivamente as potências nos terminais das antenas das estações terrenas transmissoras das redes interferida e interferente e $\ell_{1}$ e $\ell_{3 j}^{\prime}$ correspondem às atenuações de espaço livre nos lances de subida do sinal desejado e do sinal interferente, respectivamente. Ainda em

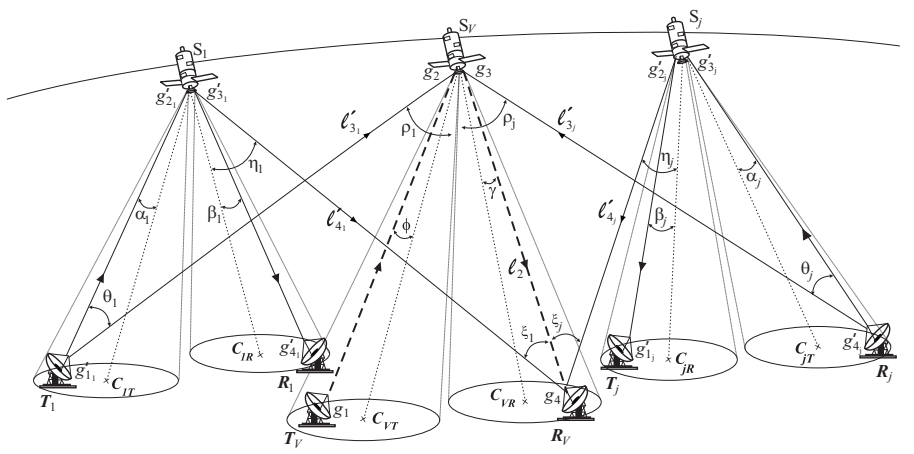

Fig. 1. Geometria utilizada no calculo de interferência envolvendo multiples redes interferentes.

(1), $g_{1 j}^{\prime}(\lambda)$ representa o ganho da antena da estação terrena transmissora interferente numa direção que forma um ângulo $\lambda$ com a a direção de apontamento da antena, $g_{1}(0)$ o ganho máximo da antena da estação terrena transmissora do enlace vítima e $g_{2}(\lambda)$ o ganho da antena receptora do satélite vítima numa direção que forma um ângulo $\lambda$ com a direção de apontamento do feixe.

Considerando-se ainda a Figura 1, observa-se que razão portadora-interferência no lance de descida relativa à interferência do sistema $S_{j}$ sobre o sistema vitima $S_{V}$ se escreve

$$
\left(\frac{C}{I}\right)_{\text {down }}=\frac{P_{3} g_{3}(\gamma) g_{4}(0) \ell_{4 j}^{\prime}}{P_{3 j}^{\prime} g_{3 j}^{\prime}\left(\eta_{j}\right) g_{4}\left(\xi_{j}\right) \ell_{2}}
$$

onde $P_{3}, P_{3 j}^{\prime}$ representam respectivamente as potências nos terminais das antenas transmissoras dos satélites das redes interferida e interferente e $\ell_{2}$ e $\ell_{4 j}^{\prime}$ correspondem às atenuações de espaço livre nos lances de descida do sinal desejado e do sinal interferente, respectivamente. Ainda em $(2), g_{3}^{\prime}(\lambda)$ representa o ganho da antena transmissora do satélite interferente numa direção que forma um ângulo $\lambda$ com a direção de apontamento do feixe, $g_{4}(\lambda)$ o ganho da antena da estação terrena receptora vítima numa direção que forma um ângulo $\lambda$ com a direção de apontamento da antena e $g_{3}(\lambda)$ o ganho da antena transmissora do satélite vítima numa direção que forma um ângulo $\lambda$ com a direção de apontamento do feixe.

Finalmente, a razão portadora-interferência total (lances de subida e descida) devida à interferência agregada das $N$ redes interferentes se escreve

$$
\left(\frac{C}{I}\right)_{t_{a g g}}=\left[\sum_{j=1}^{N}\left(\left(\frac{C}{I}\right)_{u p}^{-1}+\left(\frac{C}{I}\right)_{\text {down }}^{-1}\right)\right]^{-1}
$$

A partir de (3), (1) e (2) é possível escrever a variável $z$ que caracteriza a razão interferência - portadora (agregada) como

$$
z=\frac{I}{C}=\sum_{j=1}^{2 N} z_{j}
$$

onde

$$
z_{j}=k_{j} x_{j}
$$

com 


$$
k_{j}= \begin{cases}\frac{P_{1 j}^{\prime} g_{2}\left(\rho_{j}\right)}{P_{1}(0) g_{2}(\phi) g_{1}(0)} & j=1,2, \ldots, N \\ \frac{P_{3(j-N)}^{\prime} g_{3(j-N)}^{\prime}\left(\eta_{j-N}\right)}{P_{3} g_{3}(\gamma) g_{4}(0)} & j=N+1, \ldots, 2 N\end{cases}
$$

e com as variáveis $x_{j}$, que caracterizam os ganhos das antenas das estações terrenas transmissoras interferentes e receptora vitima, dadas por

$$
x_{j}= \begin{cases}g_{1 j}^{\prime}\left(\theta_{j}\right) & j=1,2, \ldots, N \\ g_{4}\left(\xi_{j}\right) & j=N+1, \ldots, 2 N\end{cases}
$$

Considere agora o conjunto $L$ dos indices $j$ tais que a variável $x_{j}$ corresponde a ganho nos lóbulos laterais da antena, ou seja,

$$
\begin{aligned}
L= & \left\{j \in\{1, \ldots 2 N\}: x_{j}\right. \text { corresponde a ganho } \\
& \text { nos lóbulos laterais da antena }\}
\end{aligned}
$$

Suponha que $x_{j}, j=1, \ldots, 2 N$ são variáveis aleatórias com função densidade de probabilidade dada por

$$
p_{x_{j}}\left(X_{j}\right)= \begin{cases}f_{j}\left(X_{j}\right) & j \in L \\ \delta\left(X_{j}-G_{j}\right) & j \notin L\end{cases}
$$

onde $f_{j}\left(X_{j}\right)$ são funções conhecidas e

$$
G_{j}= \begin{cases}G_{1 j}^{\prime}\left(\theta_{j}\right) & j=1,2, \ldots N \\ G_{4}\left(\xi_{j-N}\right) & j=N+1, \ldots 2 N\end{cases}
$$

Em (10), $G_{1 j}^{\prime}\left(\theta_{j}\right)$ e $G_{4}\left(\xi_{j-N}\right)$ correspondem a ganhos dados por diagramas de referência (como o da Recomendação ITU-R S.465-6 [1]), expressos como fator.

A partir de (5) e (9) tem-se

$$
p_{z j}\left(Z_{j}\right)= \begin{cases}\frac{1}{k_{j}} f_{j}\left(\frac{Z_{j}}{k_{j}}\right) & j \in L \\ \delta\left(Z_{j}-k_{j} G_{j}\right) & j \notin L\end{cases}
$$

onde $k_{j}$ e $G_{j}$ são dados por (6) e (10) respectivamente. Considerando-se que as variáveis aleatórias $z_{j}$ em (4) são estatisticamente independentes, tem-se

$$
M_{z}(v)=\prod_{j=1}^{2 N} M_{z_{j}}(v)
$$

onde $M_{z_{j}}(v)$ são as funções características das variáveis aleatórias $z_{j}$, obtidas a partir de (11), ou seja,

$$
M_{z_{j}}(v)= \begin{cases}M_{j}(v) & j \in L \\ e^{j v k_{j} G_{j}} & j \notin L\end{cases}
$$

com

$$
M_{j}(v)=\int_{-\infty}^{\infty} \frac{e^{j v Z}}{k_{j}} f_{j}\left(\frac{Z}{k_{j}}\right) d Z
$$

Substituindo-se (13) em (12) obtém-se finalmente

$$
M_{z}(v)=e^{j v K} \prod_{j \in L} M_{j}(v)
$$

onde

$$
K=\sum_{j \notin L} k_{j} G_{j}
$$

A função densidade de probabilidade da variável aleatória $z$, que caracteriza o inverso da razão portadora-interferência agregada total, pode ser obtida a partir de (15), chegando-se a

$$
p_{z}(Z)=p_{I / C}(Z)=F(Z-K)
$$

onde

$$
F(Z)=\frac{1}{2 \pi} \int_{-\infty}^{\infty}\left[\prod_{j \in L} M_{j}(v)\right] e^{-j v Z} d v
$$

com $K$ dado por (16).

Note que a razão interferência-portadora $z=I / C$ é expressa em fator. Seja $i / c$ o valor desta razão em dB. É possível verificar facilmente que as funções densidade de probabilidade das variáveis $I / C$ e $i / c$ se relacionam através da relação

$$
p_{i / c}(\alpha)=\frac{\ln 10}{10} 10^{\alpha / 10} p_{I / C}\left(10^{\alpha / 10}\right)
$$

A partir de (19) é possível obter a função distribuição de probabilidade da razão $c / i$ (expressa em $\mathrm{dB}$ ), dada por

$$
F_{c / i}(\alpha)=P\left(\frac{c}{i} \leq \alpha\right)=P\left(\frac{i}{c} \geq-\alpha\right)=\int_{-\alpha}^{\infty} p_{i / c}(\beta) d \beta
$$

\section{Caso Exponencial}

Neste caso, considera-se que os ganhos nos lóbulos laterais das antenas das estações terrenas têm função densidade de probabilidade exponenciais, ou seja,

$$
f_{j}\left(X_{j}\right)=a_{j} e^{-a_{j} X_{j}} u\left(X_{j}\right)
$$

com $u($ ) denotando a função degrau unitário. Tem-se assim, a partir de (14),

$$
M_{j}(v)=\int_{-\infty}^{\infty} \frac{e^{j v Z}}{k_{j}} a_{j} e^{-a_{j} \frac{Z}{k_{j}}} d Z=\frac{c_{j}}{c_{j}-i v}
$$

onde $c_{j}=\frac{a_{j}}{k_{j}}$ e $i=\sqrt{-1}$. Considerando-se (22), (18) se escreve

$$
F(Z)=\frac{1}{2 \pi} \int_{-\infty}^{\infty}\left[\prod_{j \in L} \frac{c_{j}}{c_{j}-i v}\right] e^{-j v Z} d v
$$

A integral em (23) pode ser calculada por resíduos sendo, em geral dada por

$$
F(Z)=\left(-\sum_{\ell=1}^{S} r_{\ell}\right) u(Z)
$$

onde $S$ é o número de singularidades da função

$$
f(x)=\left(\prod_{j \in L} \frac{c_{j}}{c_{j}-x}\right) e^{-x Z},
$$

no semi-plano direito e $r_{\ell}$ é o resíduo desta função na $\ell$-ésima singularidade. Se a função tem um polo de ordem $m$ em $x_{0}$, correspondente à $\ell$-ésima singularidade, tem-se

$$
r_{\ell}=\frac{1}{(m-1) !} \lim _{x \rightarrow x_{0}} \frac{d^{m-1}}{d x^{m-1}}\left(x-x_{0}\right)^{m} f(x)
$$




\section{Caso Gama}

Neste caso, considera-se que os ganhos nos lóbulos laterais das antenas das estações terrenas têm função densidade de probabilidade gama, ou seja,

$$
f_{j}\left(X_{j}\right)=\frac{1}{\Gamma\left(q_{j}\right)} a_{j}^{q_{j}} X_{j}^{q_{j}-1} e^{-a_{j} X_{j}} u\left(X_{j}\right)
$$

com $u($ ) denotando a função degrau unitário. Tem-se assim, a partir de (14),

$$
M_{j}(v)=\int_{-\infty}^{\infty} \frac{e^{j v Z}}{\Gamma\left(q_{j}\right)} \frac{a_{j}}{k_{j}}\left(\frac{a_{j} Z}{k_{j}}\right)^{q_{j}-1} e^{-a_{j} \frac{Z}{k_{j}}} d Z=\frac{c_{j}^{q_{j}}}{\left(c_{j}-i v\right)^{q_{j}}}
$$

onde $c_{j}=\frac{a_{j}}{k_{j}}$ e $i=\sqrt{-1}$. Considerando-se (22), (18) se escreve

$$
F(Z)=\frac{1}{2 \pi} \int_{-\infty}^{\infty}\left[\prod_{j \in L} \frac{c_{j}^{q_{j}}}{\left(c_{j}-i v\right)^{q_{j}}}\right] e^{-j v Z} d v
$$

Conforme indicado em [9], a integral em (29) pode ser calculada utilizando-se a série

$$
F(Z)=C \sum_{k=0}^{\infty} \delta_{k} \frac{Z^{\rho+k-1}\left(c^{*}\right)^{\rho+k}}{\Gamma(\rho+k)} e^{-c^{*} Z} ; Z>0
$$

onde $\rho=\sum_{j \in L}\left(q_{j}\right), c^{*}=\max _{j \in L}\left(c_{j}\right)$ e $C=\prod_{j \in L}\left(c_{j} / c^{*}\right)^{q_{j}}$.

Em (30), os coeficientes $\delta_{k}$ são dados por

$$
\delta_{k+1}=\frac{1}{k+1} \sum_{i=1}^{k+1} i \gamma_{i} \delta_{k+1-i} \quad ; \quad k=0,1, \ldots
$$

$\operatorname{com} \delta_{0}=1$ onde

$$
\gamma_{k+1}=\sum_{j \in L} \frac{q_{j}\left(1-\frac{c_{j}}{c^{*}}\right)^{k}}{k} \quad ; \quad k=1,2, \ldots
$$

A convergência da série em (30) é demostrada em [9].

\section{RESUltAdOS NumÉRICOS}

Nesta seção, os resultados analíticos obtidos na Seção II para os casos exponencial e gama são aplicados a situações onde sistemas de satélites geoestacionários compartilham uma mesma faixa de freqüências. Mais especificamente, são analisados três cenários envolvendo, respectivamente, dois, três e cinco sistemas multi-feixe operando na Banda Ka. Nestes sistemas a comunicação é feita utilizando-se dois tipos de enlace: enlaces diretos (forward link), correspondentes a transmissões das estações gateway para os terminais de usuários, e enlaces reversos (return link), correspondentes a transmissões dos terminais de usuários para as estações em gateway. Nos cenários analisados, os sistemas envolvidos foram considerados homogêneos. Os parâmetros de enlace desses sistemas são apresentados na Tabela I para os enlaces direto e reverso. As áreas de serviço desses sistemas são definidas por múltiplos feixes circulares com 0.7 graus de abertura e diagrama de radiação dados pela Recomendação ITU-R S.672 [10] (com $L_{N}=-15 \mathrm{~dB}, z=1, a=2.58, b=6.32$ e $\left.\alpha=1.5\right)$ e com re-uso de frequência do tipo "quatro cores". O cenário mais complexo
(Cenário III) envove um abiente interferente contendo um sistema vítima (na posição oirbital $23^{\circ} \mathrm{W}$ ) e quatro sistemas interferentes $\left(27^{\circ} \mathrm{W}, 25^{\circ} \mathrm{W}, 21^{\circ} \mathrm{W}\right.$ e $\left.19^{\circ} \mathrm{W}\right)$. Os percursos das interferências dos lances de subida e descida são apresentados na Figura 2. Conforme ilustrado nesta figura, os enlaces interferentes estão associados a feixes de recepção e transmissão dos satélites que correpondem aos vizinhos mais próximos dos feixes (de recepção e transmissão do satélite) correpondentes ao enlace vítima. No Cenário II, são considerados o sistema vítima $\left(23^{\circ} \mathrm{W}\right)$ e e dois sistemas interferentes $\left(25^{\circ} \mathrm{W}\right.$ e $\left.21^{\circ} \mathrm{W}\right)$ e no Cenário I, o sistema vítima $\left(23^{\circ} \mathrm{W}\right)$ sofre interferência de apenas um sistema $\left(25^{\circ} \mathrm{W}\right)$. Em todos os cenários, as estações terrenas transmissoras dos sistemas interferentes são colocadas nas posições mais desfavoráveis (em termos de interferência) de seus feixes de cobertura. As demais estações terrenas foram coloacadas no centro de seus feixes.

TABELA I

PARÂMETROS dos ENLACES

\begin{tabular}{||l|r|r||}
\hline \multicolumn{1}{|c|}{ PARÂMETRO } & $\begin{array}{c}\text { Enlace } \\
\text { Direto }\end{array}$ & $\begin{array}{c}\text { Enlace } \\
\text { Reverso }\end{array}$ \\
\hline \hline Lance de subida: & 29.5 & 28.75 \\
frequência [GHz] & 13 & 1.2 \\
diâmetro da antena da estação terrena transmissora [m] & 48.78 \\
ganho máximo da antena da estação terrena transmissora [dBi] & 69.25 & 23 \\
potência da estação transmissora [dBW] & 13 & 16.02 \\
margem para atenuação por chuva [dB] & 3 & 10 \\
atenuação atmosférica [dB] & 500 & 500 \\
temperatura de ruido do satélite [K] & 47.97 & 51.47 \\
ganho máximo da antena de receptor do satélite [dBi] & & \\
\hline Lance de descida: & 19.2 & 18.7 \\
frequência [GHz] & 1.2 & 13 \\
diâmetro da antena da estação terrena receptora [m] & 45.05 & 65.52 \\
ganho máxima da antena da estação terrena receptora [dBi] & 21.14 & 18 \\
potência em receptor do satélite [dBW] & 3 & 3 \\
back-off [dB] & 10.84 & 8 \\
margem para atenuação por chuva [dB] & 3 & 3 \\
atenuação atmosférica [dB] & 241 & 26914 \\
temperatura de ruido do receptor [K] & 51.47 & 47.97 \\
ganho máximo da antena de transmissão do satélite [dBi] & & \\
\hline \hline
\end{tabular}

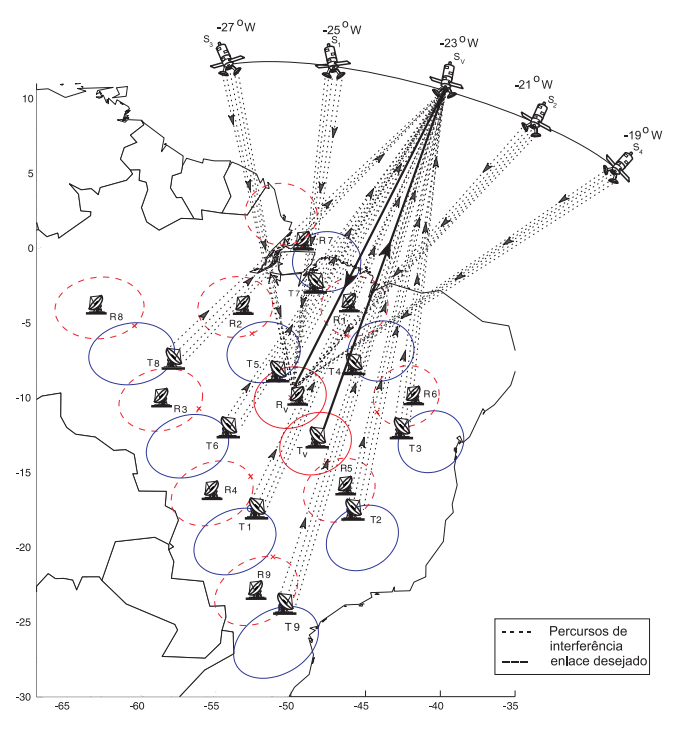

Fig. 2. Percursos de interferência correspondentes ao Cenário III

Os ganhos $G_{1 j}^{\prime}()$ e $G_{4}()$ que aparecem em (10) foram tomados como os do diagrama de referência em [1], ou seja,

$$
G_{1 j}^{\prime}(\lambda)=G_{4}(\lambda)=10^{3,2} \lambda^{-2,5}
$$


No Caso Exponencial, os parâmetros $a_{j}$ que aparecem em (21) foram determinados de modo que a probabilidade do ganho $x_{j}$ exceder o ganho $G_{j}$ em (10) seja igual a 0,1 . No Caso Gama, além desta condição, foram considerados valores específicos da razão desvio padrão-média (RDPM) na determinação dos parâmetros $a_{j}$ e $q_{j}$ que aparecem em (27). Note que RDPM=1 corresponde ao caso exponencial. Os resultados para os três cenários considerados são apresentados nas figuras 3 a 5 .

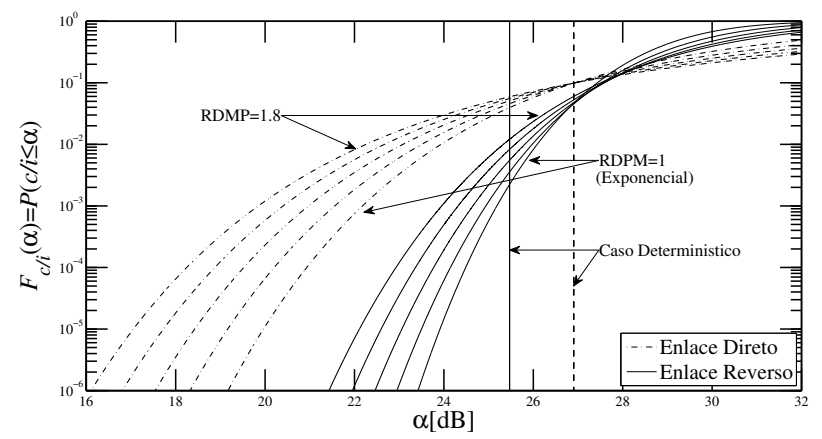

Fig. 3. Função distribuição de probabilidade da razão portadora-interferência (caso exponencial, enlace direto).

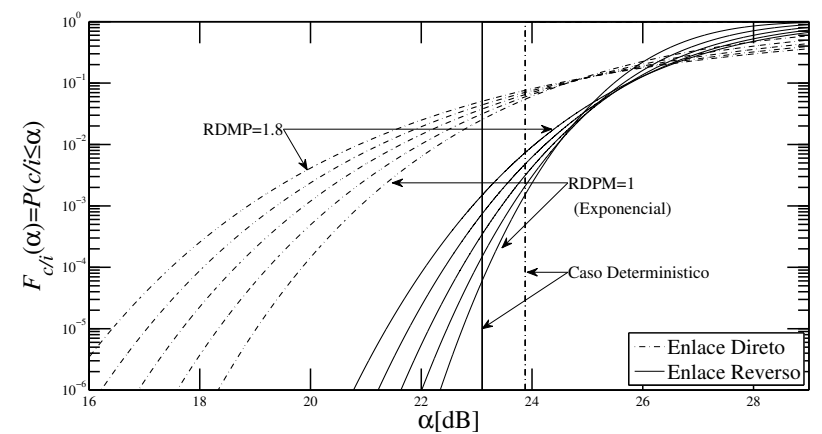

Fig. 4. Função distribuição de probabilidade da razão portadora-interferência (caso exponencial, enlace reverso).

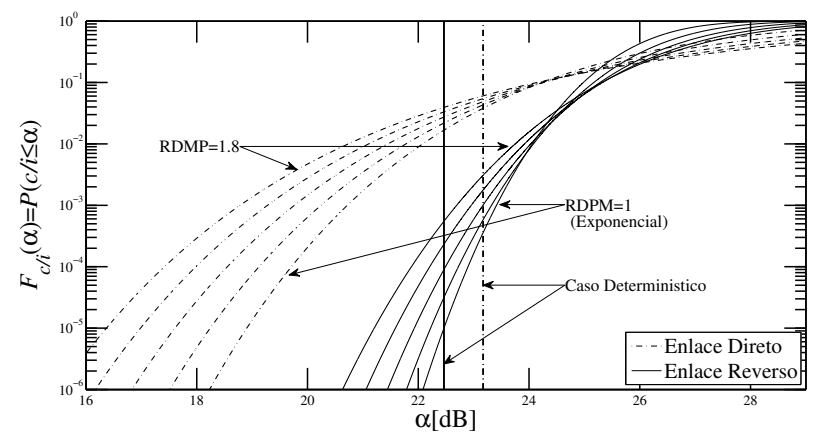

Fig. 5. Função distribuição de probabilidade da razão portadora-interferência (caso gama, enlace direto).

\section{CONCLUSÕES}

Comparada ao cálculo determinístico tradicional, que utiliza diagramas de referência, a modelagem probabilística apresentada descreve de maneira mais realista os ganhos nos lóbulos laterais das antenas envolvidas. Além disso, ela permite determinar analiticamente o comportamento estatístico da razão portadora-interferência, possibilitando, entre outros pontos, a quantificação da probabilidade da razão portadorainterferência ser inferior ao valor calculado pelo método determinístico. Conforme esperado, esta probabilidade diminui à medida que o número de parcelas interferentes aumenta. Este fato pode ser melhor observado nas figuras 3 a 5 , que apresentam, respectivamente as funções distribuição de probabilidade da razão $c / i$ correspondentes aos três cenários considerados. No caso do enlace reverso e da modelagem expenencial para os ganhos das antenas observa-se, por exemplo, que a probabilidade da razão portadora-interferência ser inferior ao valor calculado pelo método determinístico é igual a $2,2 \times 10^{-3}$ no Cenário I, $6 \times 10^{-5}$ no Cenário II e $10^{-5}$ no Cenário III.

Estas figuras mostram ainda que, caso o valor da razão portadora-interferência calculado pelo método determinístico não seja inferior ao valor requerido de $c / i$, a modelagem probabilística permite uma avaliação da probabilidade com a qual este valor requerido não é atendido. Observa-se, por exemplo, que no Cenário III, Enlace Reverso (ver Figura 5) o valor de $24 \mathrm{~dB}$ para a razão portadora-interferência não é atendido quando o cálculo determinístico é utilizado. Por outro lado, o cálculo probabilístico indica que a probabilidade deste valor não ser atendido varia de aproximadamente $7 \times 10^{-3}$ $(\mathrm{RDPM}=1)$ até $1,8 \times 10^{-2}(\mathrm{RDPM}=1,8)$, o que implica emn probabilidades do valor requerido de $c / i$ ser atendido que variam respectivamente de 0,993 a 0,982. Nota-se ainda que as diferenças entre a modelagem proposta e a modelagem determinística são mais acentuadas no enlace reverso. Isto acontece devido às características particulares dos sistemas satélite considerados (multi-feixe). Observa-se, por exemplo, que no enlace direto, a interferência no lance de descida, além de ser dominante, tem um número de parcelas bem menor que o número de parcelas correspondentes à interferência no lance de subida.

\section{REFERÊNCIAS}

[1] Reference Earth Station Radiation Patterns for Use in Coordination and Interference Assessment in the Frequency Range from 2 to about $30 \mathrm{GHz}$, Recommendation ITU-R S.465-6, Genebra, 2010.

[2] Proposed Amendment to Report 391-2: Some statistical Properties od Antenna Sidelobes, Documento CCIR 4/32, submetido pela administração do Japão à Reunião do CCIR, Genebra, 1976.

[3] Jeruchim M. C., A Statistical Approach to Satellite Interference Levels, Records of the International Conference on Commuunications, pp 35.3.1-353.4, Toronto 1978.

[4] Karmel P. R., Statistical Properties of Antenna Sidelobes, Comsat Technical Review, Vol. 9, No. 1, pp 91-120, 1979.

[5] Statistical Model for Sidelobe Interference in Satellite Networks, Relatório Final, Contrato INTEL-198, Maio 1982.

[6] Kadrichu A., Prasana S., Mukunda R., Stochastic Evaluation of Interference Aggregation in Satellite Communications, Proceedings of the IEEE International Communucations Conference, pp. 50.1.1-50.1.7., Seattle, Junho 1987

[7] Albuquerque J. P., Fortes J. M., Statistical Aspects in the Evaluation of Interference among Satellite Networks, Proceedings of the IEEE International Communucations Conference, pp. 7.2.1-7.2.5, Philadelphia, 1988

[8] Albuquerque J. P., Fortes J. M., Gouveia W. P., Uso mais eficiente da órbita de satélites geoestacionários através do tratamento probabilístico da interferência entre redes, Anais do VII Simpósio Brasileiro de Telecomunicações, pp 95-101, Florianópolis, Setembro 1989.

[9] Moschopoulos, P. G., The distrubution of the sum of independent gamma random variables, Annals of the Institute of Statistical Mathematics, Vol. 37, No. 3, pp 541-544, 1985.

[10] Satellite antenna radiation pattern for use as a design objective in the fixedsatellite service employing geostationary satellites, Recommendation ITU-R S.672, Genebra, 1997. 